\section{CardioRenal Medicine}

\title{
The ADMA-Metformin Hypothesis: Linking the Cardiovascular Consequences of the Metabolic Syndrome and Type 2 Diabetes
}

\author{
William H. Bestermann, Jr. \\ Vascular Medicine Center, Holston Medical Group, Kingsport, Tenn., USA
}

\section{Key Words}

ADMA $\cdot$ Asymmetric dimethylarginine $\cdot$ Diabetes, type $2 \cdot$ Hyperlipidemia $\cdot$ Hypertension

Insulin resistance $\cdot$ Metabolic syndrome $\cdot$ Metformin

\begin{abstract}
Metformin and asymmetric dimethylarginine (ADMA) are structural analogs. They have opposite effects at multiple points on complex signaling pathways that coordinate energy, molecular synthesis, growth, and metabolism with nutrient intake. Excess saturated fats and glucose may initiate the methylation of arginine residues in proteins involved in the transcription of genes mediating inflammation, cell proliferation, apoptosis, and oncogenesis. Free ADMA may appear in the circulation after proteolysis of these proteins when the work of transcription is complete and ADMA subsequently functions as a signaling molecule. In children, ADMA levels are not significantly related to the usual metabolic syndrome risk factors but instead there is a significant association between ADMA and alkaline phosphatase - a marker of normal growth. There is only one direct study that shows that ADMA negates the metabolic effects of metformin. There are no investigations that demonstrate that metformin blocks the effect of ADMA and so this review must be considered hypothesis generating. The potential implications of the metformin-ADMA relationship merit further investigation.

Copyright $\odot 2011$ S. Karger AG, Basel
\end{abstract}

\section{Introduction}

Asymmetric dimethylarginine (ADMA), a guanidine-substituted analog of L-arginine, competitively inhibits the conversion of L-arginine to nitric oxide (NO) and citrulline. Elevated ADMA levels are associated with many cardiovascular risk factors, including age, hy- 


\section{CardioRenal Medicine}

\begin{tabular}{l|l}
\hline Cardiorenal Med 2011;1:211-219 \\
\hline DOI: 10.1159/000332382 & $\begin{array}{l}\text { @ 2011 S. Karger AG, Basel } \\
\text { www.karger.com/crm }\end{array}$ \\
\hline
\end{tabular}

Bestermann: The ADMA-Metformin Hypothesis

pertension, diabetes, insulin resistance, hypercholesterolemia, hypertriglyceridemia, and hyperhomocysteinemia. For a number of these conditions, there is evidence of a causal relationship. Virtually all of the traditional cardiovascular risk factors are associated with endothelial vasodilator dysfunction, an early and critical event in the pathogenesis of cardiovascular disease states. Endothelial dysfunction is thought to contribute to atherosclerotic plaque formation, plaque progression, and ultimately plaque rupture, as do many of the traditional risk factors. These facts have led to speculations that diverse risk factors ultimately share common pathophysiological pathways. ADMA has been described as a ubiquitous mechanism of endothelial pathobiology shared by all risk factors and markers examined to date'. A large body of literature supports 'ADMA as an "Über marker", a biochemical factor mediating the adverse vascular effects of many other risk factors and markers' [1].

In addition to its effects on the vasodilator portion of endothelial function, NO inhibits key processes involved in arterial disease, including leukocyte adhesion, vascular smooth muscle cell proliferation, and platelet aggregation. In animal models, changes in vascular NO synthesis have a powerful impact on the progression of atherosclerosis. Impaired NO-dependent endothelial function independently predicts the incidence of cardiovascular events. Major causes of impairment of NO-dependent endothelial function are endogenous inhibitors of NO production, the most important of which is ADMA [1].

\section{Endothelial Dysfunction and Insulin Resistance Are Tightly Linked}

Multiple studies suggest that endothelial dysfunction and insulin resistance are linked. Reduced NO production is a critical factor in endothelial dysfunction [2] and seems to play an early role in the development of insulin resistance [3]. Several lines of evidence indicate that endothelial function is abnormal in circumstances in which sensitivity to endogenous insulin is reduced [4]. In rats, the development of hypertension related to the infusion of the NO synthase (NOS) inhibitor L-NMMA ( $N^{G}$-monomethyl-L-arginine) and reduction of NO production is associated with impaired glucose metabolism [5], and insulin resistance is not present in patients with many of the secondary forms of hypertension [6], suggesting a common cause for metabolic and hemodynamic abnormalities in the metabolic syndrome. In endothelial NOS (eNOS)-/- mice, insulin stimulation of muscle blood flow was impaired and strongly related to impaired stimulation of muscle glucose uptake [7].

Plasma ADMA can increase rapidly in response to excess nutrient levels. A single highfat meal may more than double circulating ADMA concentrations in diabetic patients within $5 \mathrm{~h}$ and then ADMA may participate in a vicious cycle of oxidative particle production [8, 9]. In rabbits with alloxan-induced diabetes, ADMA levels were increased in proportion to the increase in plasma glucose [10]. Importantly, ADMA levels are tripled in rats with noninsulin-dependent diabetes mellitus [11].

\section{Endothelial Dysfunction Is Only Part of the ADMA Story}

Although the role of ADMA as an inhibitor of NO production and endothelial function is well established, many questions can be raised: Why would a high-fat meal or a glucose challenge raise ADMA levels so promptly and what purpose would it serve? Furthermore, that ADMA effects are mediated by ADMA competition with L-arginine in the production of $\mathrm{NO}$ is problematic, and has been termed 'the arginine paradox'. ADMA levels found in health $(500 \mathrm{nmol} / \mathrm{l}$ to $1.2 \mu \mathrm{mol} / \mathrm{l}$ ) or many disease states (up to $3 \mu \mathrm{mol} / \mathrm{l}$ ) are too low to competitively inhibit the conversion of L-arginine to NO. Plasma L-arginine concentrations are 


\section{CardioRenal Medicine}

Cardiorenal Med 2011;1:211-219

DOI: $10.1159 / 000332382$

Bestermann: The ADMA-Metformin Hypothesis

on the order of $30-100 \mu \mathrm{mol} / \mathrm{l}$ and the intracellular levels of $\mathrm{L}$-arginine may be as high as $2 \mathrm{mmol} / \mathrm{l}$ [12]. With such an excess of L-arginine, ADMA should be inert but 'experimental evidence shows that even very low concentrations of the methylarginines have dramatic effects'. Infusions of ADMA into healthy volunteers produce rapid increases in blood pressure and vascular resistance with a fall in cardiac output and heart rate. Plasma concentrations achieved in the infusion were comparable to those found in pathophysiological conditions $[13,14]$.

Indeed, there is convincing evidence that the long-term vascular effects of ADMA are not solely mediated by inhibition of endothelial NO synthesis. Long-term infusion of ADMA into mice raised blood pressure and produced coronary lesions manifested by medial thickening and perivascular fibrosis to an equal extent in wild-type and eNOS-knockout mice and did not reduce plasma or urine NO metabolite concentrations [15].

\section{Circulating ADMA Levels Vary Quantitatively with the Degree of Insulin Resistance}

The only established role of ADMA is the inhibition of NO production by the three recognized forms of NOS - eNOS, inducible nitric oxide synthase (iNOS), and neuronal NOS. Most of the literature concerns eNOS. The cardiovascular biology of the ADMA metabolic pathways was recently summarized: 'Although it is unnecessary to invoke additional targets for ADMA, it is worth noting that many microbes produce ADMA and express the enzymes necessary to metabolize ADMA, yet do not express NOS' [16]. This observation suggests some additional unrecognized action of ADMA [17].

An important recent study extends the link between insulin resistance and endothelial dysfunction. The association of plasma ADMA with insulin resistance was greater than that with steady-state plasma glucose concentrations (the specific determinant of insulin resistance) and fasting plasma insulin (a commonly used surrogate estimate of insulin resistance) [18]. Steady-state plasma glucose concentrations were a strong predictor of ADMA levels, and the relationship between insulin resistance and circulating ADMA was independent of other factors associated with insulin resistance. Plasma ADMA concentrations were positively correlated with impairment in insulin-mediated glucose disposal in non-diabetic, normotensive subjects $(\mathrm{r}=0.73 ; \mathrm{p}<0.001)$. The authors concluded that the close correlation of circulating ADMA with insulin resistance may provide a more general explanation for elevated plasma ADMA concentrations in patients with type 2 diabetes, essential hypertension, and renal failure. An increase in the prevalence of insulin resistance is well documented in patients with type 2 diabetes and essential hypertension. Plasma ADMA levels were not increased in hypertensive patients unless they were also insulin resistant [19]. ADMA levels correlate with both endothelial dysfunction and the level of insulin resistance. This tight correlation between ADMA levels and insulin resistance suggests that ADMA participates in signaling pathways important in the determination of the metabolic syndrome.

\section{Metformin Mechanism of Action Involves ADMA Blockade at Multiple Levels of Function}

Metformin, employed clinically in the treatment of type 2 diabetes, lowers insulin resistance and oxidative stress, and improves endothelial function. The prevailing thinking regarding the mechanism of action of metformin points to the activation of adenosine monophosphate-activated protein kinase (AMPK) [20]. In fact, the effects of metformin on he- 


\section{CardioRenal Medicine}

Cardiorenal Med 2011;1:211-219

DOI: $10.1159 / 000332382$

2011 S. Karger AG, Base www.karger.com/crm

Fig. 1. Chemical structures for ADMA and the anti-diabetic agent metformin. The two chemicals are structural analogs and these agents may be competitive antagonists in vascular smooth muscle cells to regulate insulin resistance.

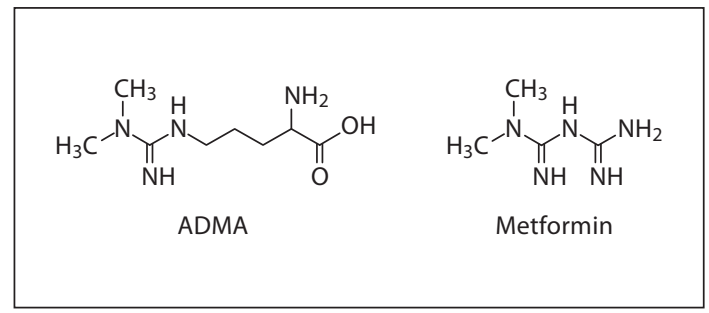

patic glucose neogenesis are preserved in AMPK-knockout mice [21] and so it may be useful to think of this medication in a broader context.

Metformin, a structural analog of ADMA (fig. 1), has beneficial effects on the multiple abnormalities associated with insulin resistance. In every instance, these associations are the opposite of those found with ADMA. Metformin decreases hyperglycemia, improves diastolic function, decreases total cholesterol, decreases low- and very-low-density lipoprotein cholesterol levels, increases high-density lipoprotein cholesterol levels, decreases oxidative stress, improves vascular relaxation, decreases plasminogen activator inhibitor levels (PAI1), increases tissue plasminogen activator activity, and decreases von Willebrand factor levels, platelet aggregation and adhesion, and free fatty acid levels [22].

Since metformin is a structural analog of ADMA and these compounds have opposing effects on the metabolic features of insulin resistance, it is tempting to speculate that these two compounds may function as competitive antagonists. However, the available literature almost exclusively deals with the activity of ADMA as a competitive antagonist of L-arginine in NO formation. It is unlikely that a competitive antagonist would compete with a competitive antagonist, but other sites of competition may impact important signaling pathways.

Metformin has favorable effects on virtually every component of the metabolic syndrome. Known metabolic effects of metformin involve mitochondrial pathways and other downstream effects. Detaille et al. [23] showed that intact Xenopus laevis oocytes exposed to metformin demonstrated a $40 \%$ reduction in the rotenone-sensitive activity of respiratory chain complex I in mitochondria. This effect was demonstrated only in intact incubated oocytes and not in mitochondria isolated by centrifugation, suggesting an important role for intact membranes. Drug injected directly into oocytes also had no effect. Metformin transport was hindered and the inhibition of complex 1 totally disappeared when its structural analog ADMA was placed together with metformin. These data support the view that metformin may recognize specific membrane sites, likely belonging to effector systems, before penetrating the cell in a bound state via a putative endocytic event. ADMA competitively inhibits the activity of its structural analog metformin at the membrane level. While this study is the only direct investigation of the antagonism of metformin and ADMA, multiple articles show that metformin and ADMA have opposite effects in important signaling pathways. ADMA may be the agonist and metformin the ADMA antagonist, blocking the effect of ADMA as a signaling molecule.

There is evidence of important effects of ADMA in the mitochondria of pulmonary artery endothelial cells. ADMA added to these cells stimulates the redistribution of eNOS from the plasma membrane to the mitochondrion where ADMA uncouples eNOS and causes a shift from NO to peroxynitrite production. Uncoupled eNOS located in the mitochondrion results in an increase in nitrated proteins in the mitochondria. Taken together, these events lead to an increase in oxidative stress in the mitochondria with subsequent mitochondrial dysfunction and reduced ATP production [24]. 


\section{CardioRenal \\ Medicine}

\begin{tabular}{l|l}
\hline \multicolumn{2}{l}{ Cardiorenal Med 2011;1:211-219 } \\
\hline DOI: 10.1159/000332382 & $\begin{array}{l}\text { @ 2011 S. Karger AG, Basel } \\
\text { www.karger.com/crm }\end{array}$ \\
\hline
\end{tabular}

Bestermann: The ADMA-Metformin Hypothesis

\section{ADMA Signaling Modulates Cellular Growth, Differentiation, Proliferation, Inflammation, Cell Survival, and Apoptosis}

The diverse effects of ADMA and their potential neutralization by metformin may best be understood in the context of epigenetic changes produced by arginine methylation followed by proteolytic degradation of transcriptional proteins to produce free ADMA, a molecule participating in complex signaling cascades in the circulation and cytoplasm. Very interestingly, in children ADMA is not correlated with obesity or cardiovascular risk factors; rather it is tightly correlated with normal growth [25]. Epigenetics is defined as heritable alterations in gene expression that are not encoded in the DNA sequence itself. Epigenetic gene expression occurs as a result of post-translational modification of DNA and histone proteins on the chromosome. Inactive genes exist in a tightly compacted state termed heterochromatin. Methylation of coactivator complex proteins combined with histone methylation contribute to moving heterochromatin to euchromatin, which 'opens' the chromosome to allow gene activation and transcription [26]. Even though epigenetic changes do not involve the fixed DNA code, they are transmissible to the next generation [27].

ADMA is produced as a result of arginine methylation primarily via a protein methyltransferase (PRMT-1) with subsequent proteolytic degradation and release of ADMA into the cytoplasm and circulation. But targets of protein methylation are not just any proteins. Protein arginine methylation modulates transcription, RNA metabolism, and protein-protein interactions that control histone function, cellular differentiation, signal transduction, proliferation, survival, inflammation, and apoptosis. Protein arginine methylation and circulating ADMA may be important components of the pathophysiology of the metabolic syndrome, atherosclerotic vascular disease, cardiomyopathy, and pulmonary and neoplastic diseases [28].

As mentioned earlier, ADMA levels are elevated within minutes after fat or glucose ingestion. Excess ingestion of these nutrients hypothetically could activate arginine methylation releasing ADMA into the circulation. Vascular smooth muscle cells from diabetic mice demonstrated persistently elevated inflammatory responses that were still present after exposure to high glucose. Increased expression of these inflammatory markers remained even after these cells were removed from the mice and placed in tissue culture. These epigenetic changes represent a plausible mechanism of metabolic memory. These findings may also explain why diabetic vascular complications and inflammation persist even after achieving glycemic control and why early glycemic control may have legacy effects [29].

When the work of transcription is completed, and asymmetrically methylated arginine residues are released into the cytoplasm and circulation by proteolysis, circulating ADMA may activate multiple signaling cascades. Studies of ADMA effects on gene expression in human coronary artery endothelial cells demonstrated significant increases in the activity of genes involving cell growth and/or maintenance, the cell cycle, and cellular proliferation [30].

\section{ADMA Increases the Production of Reactive Oxygen Species}

ADMA increases angiotensin II leading to additional reactive oxygen species (ROS) formation and growth factor activation [31-33]. In obesity, aldosterone levels are relatively elevated and contribute to the development of the metabolic syndrome [34]. Activation of the mineralocorticoid receptor by aldosterone leads to the production of ROS primarily by the mitochondria through inhibition of respiratory complex I (fig. 2) [35].

Taken together, these studies indicate that multiple cardiometabolic pathways converge to increase oxidative stress, which may be an important common route to the production of cardiovascular disease and events. 


\section{CardioRenal Medicine}

Cardiorenal Med 2011;1:211-219

DOI: $10.1159 / 000332382$

2011 S. Karger AG, Basel www.karger.com/crm

Bestermann: The ADMA-Metformin Hypothesis
Fig. 2. Aldosterone transactivates the epidermal growth factor and related signaling cascades that impact multiple chronic conditions [35]. S6K1 activation degrades IRS-1 contributing to insulin resistance [37] and may contribute to oncogenesis [38]. ADMA increases ROS and should have a similar effect [33]. EGFR = Epidermal growth factor receptor; TSC1/2 = tuberous sclerosis complex.

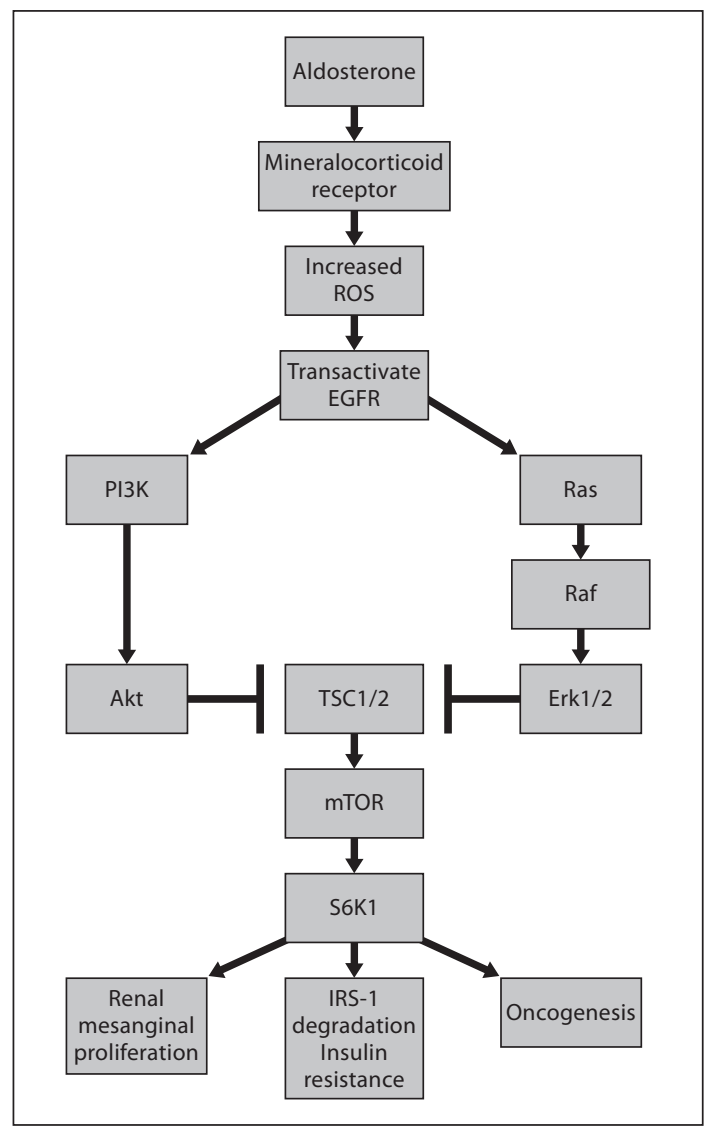

ADMA increases superoxide and peroxynitrite levels by inhibiting arginine conversion to NO, uncoupling NOS and increasing iNOS. Superoxide and peroxynitrite subsequently transactivate the epidermal growth factor receptor upregulating the phosphoinositide 3-kinase (PI3K)/Akt1 and rat sarcoma (Ras)/RAF proto-oncogene serine/threonine-protein kinase (Raf)/extracellular signal-regulated kinase (Erk1/2) cascades which converge at mammalian target of rapamycin (mTOR). mTOR and AMPK [36] are linked metabolic hubs that integrate nutrient intake and energy balance with growth, proliferation, and metabolism. Nutrients reduce AMPK activity resulting in a shift to synthesis of cholesterol and triglycerides, and increased mTOR effects. mTOR upregulates p70 ribosomal protein S6 kinase 1 (S6K1) which in turns degrades insulin receptor substrate (IRS-1) resulting in insulin resistance [37] and contributing to oncogenesis [38]. ADMA and oxidants also activate Toll-like receptor (TLR4; fig. 3) [39].

These signaling pathways increase the activity of multiple inflammatory mediators, including interleukin (IL)-1 $\beta$, IL-6, IL-8, p38 mitogen-activated protein kinase (p38MAPK), cJun N-terminal kinase (JNK), tumor necrosis factor- $\alpha$, nuclear factor (NF)- $\kappa$ B, and iNOS [40].

\section{Metformin and ADMA Have Opposite Effects on Cardiometabolic Pathways}

Metformin reduces the size of myocardial infarction in ischemia-reperfusion studies in diabetic and non-diabetic laboratory animals. Part of the mechanism of the myocardial protection involved increased AMPK-induced phosphorylation of eNOS at 1177. Metformin did 


\section{CardioRenal Medicine}

\begin{tabular}{l|l}
\hline Cardiorenal Med 2011;1:211-219 \\
\hline DOI: 10.1159/000332382 & $\begin{array}{l}\text { @ 2011 S. Karger AG, Basel } \\
\text { www.karger.com/crm }\end{array}$ \\
\hline
\end{tabular}

Bestermann: The ADMA-Metformin Hypothesis
Fig. 3. ADMA activates TLR4 via ROS [39]. TLR4 activates multiple inflammatory pathways [40]. TSC1/2 = Tuberous sclerosis complex.

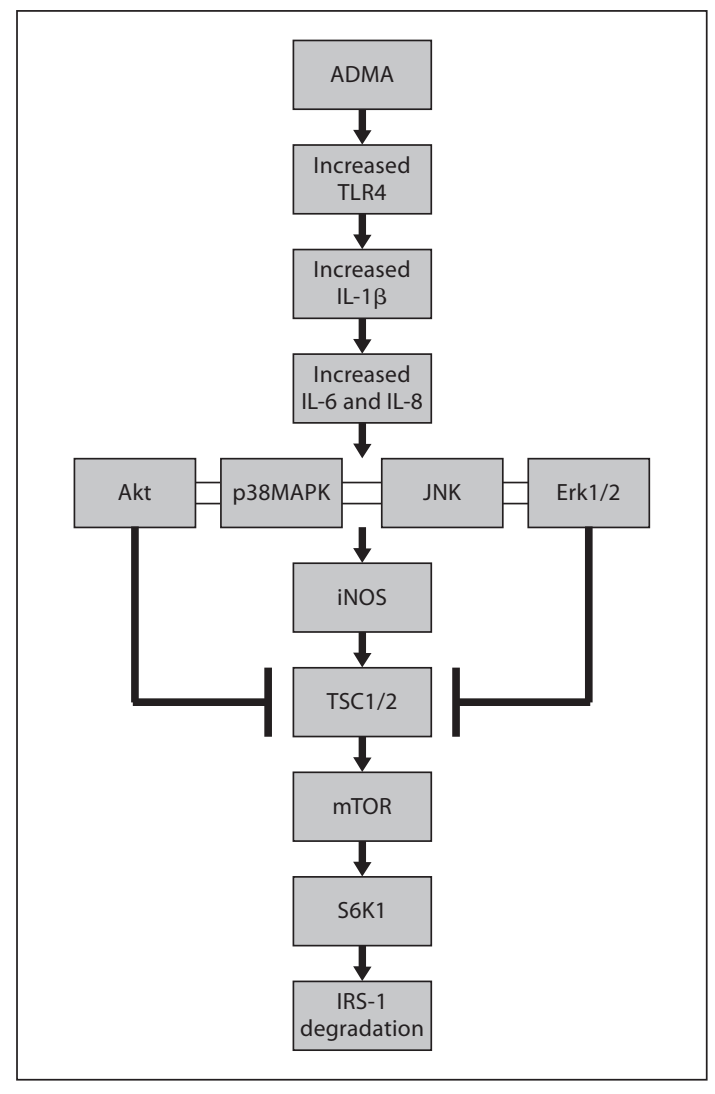

not reduce infarction size in eNOS-/- mice, suggesting that eNOS and NO play an important role in the cardioprotective effects of metformin [41].

Incubation of human pancreatic islet cells from patients with type 2 diabetes with metformin resulted in a return of protein kinase $\mathrm{C}(\mathrm{PKC})$ and nitrotyrosine levels towards control values [42]. Metformin dose dependently diminished the IL-1 $\beta$-induced release of the proinflammatory cytokines IL-6 and IL-8 in endothelial cells, smooth muscle cells, and monocytes. It diminished the activation of Akt, Erk, JNK, PKC, and p38MAPK in vascular smooth muscle cells, and dose-dependently inhibited NF- $\kappa$ B induction [43]. Metformin produces cell cycle arrest in the $\mathrm{G}_{0} / \mathrm{G}_{1}$ phase of the cell cycle and it inhibits mTOR directly [44]. Patients with type 2 diabetes on metformin have a lower risk of cancer [45].

\section{Conclusions}

Abnormally activated pathways of growth, proliferation, inflammation, and apoptosis constitute a common denominator of multiple chronic cardiovascular conditions. Metformin may interfere with these pathways by blocking ADMA entry into the cell at the level of cationic transport. This may represent an entirely new understanding of the mechanism of action of metformin and help to explain its beneficial effect on cardiometabolic risks and outcome. Still, there is only one direct study that shows that ADMA negates the metabolic effects of metformin. There are no investigations that demonstrate that metformin blocks the effect of ADMA and so this review must be considered hypothesis generating. The potential implications of the metformin-ADMA relationship merit further investigation. 


\section{CardioRenal Medicine}

Cardiorenal Med 2011;1:211-219

DOl: 10.1159/000332382

Bestermann: The ADMA-Metformin Hypothesis

\section{References}

1 Cooke JP: Asymmetric dimethyarginine: the Uber marker. Circulation 2004;109:1813-1819.

2 Cooke JP: The endothelium: a new target for therapy. Vasc Med 2000;5:49-53.

3 Sydow K, Mondon CE, Cooke JP: Insulin resistance: potential role of the endogenous nitric oxide inhibitor ADMA. Vasc Med 2005; 10(suppl 1):S35-S43.

4 Balletshofet BM, Ritting K, Enderle MD, et al: Endothelial dysfunction is detectable in young normotensive first-degree relatives of subjects with type 2 diabetes in association with insulin resistance. Circulation 2000;101:1780-1784.

5 Baron AD, Zhu JS, Marshall S, et al: Insulin resistance after hypertension induced by the nitric oxide synthesis inhibitor L-NMMA in rats. Am J Physiol 1995;269:E709-E715.

-6 Marigliano A, Tedde R, Sechi LA: Insulinemia and blood pressure. Relationships in patients with primary and secondary hypertension, and with or without glucose metabolism impairment. Am J Hypertens 1990;3:521-526.

7 Duplain H, Burcelin R, Sartori C, et al: Insulin resistance, hyperlipidemia, and hypertension in mice lacking endothelial nitric oxide synthase. Circulation 2001;104:342-345.

8 Sydow K, Munzel T: ADMA and oxidative stress. Atherosclerosis Suppl 2003;4:41-51.

-9 Fard A, Tuck CH, Donis JA, et al: Acute elevations of plasma asymmetric dimethylarginine and impaired endothelial function in response to a high-fat meal in patients with type 2 diabetes. Arterioscler Thromb Vasc Biol 2000;20:20392044.

10 Masuda H, Goto M, Tamaoki S: Accelerated intimal hyperplasia and increased endogenous inhibitors for NO synthesis in rabbits with alloxan-induced hyperglycemia. Br J Pharmacol 1999;126:211-218.

-11 Lin KY, Akira I, Asagami R, Tsao PS, Adimoolam S, Kimoto M, Reaven GM, Cooke JP: Impaired nitric oxide pathway in diabetes mellitus. Circulation 2002;106:987-992.

12 Wu G, Morris SM Jr: Arginine metabolism: Nitric oxide and beyond. Biochem J 1998;336:1-17.

13 Achan V, Broadhead M, Malaki M: Asymmetric dimethylarginine causes hypertension and cardiac dysfunction in humans and is actively metabolized by dimethylarginine dimethylaminohydrolase. Arterioscler Throm Vasc Biol 2003;23:1455-1459.

14 Kielstein JT, Impraim B, Samuel S, et al: Cardiovascular effects of systemic nitric oxide synthase inhibition with asymmetric dimethylarginine in humans. Circulation 2004;109:172-177.

15 Suda O, Tsutsui M, Morishita T, et al: Asymmetric dimethylarginine produces vascular lesions in endothelial nitric oxide synthase-deficient mice: involvement of renin-angiotensin system and oxidative stress. Arterioscler Thromb Vasc Biol 2004;24:1682-1688.

16 Santa Maria J, Vallance P, Leiper J: Identification of microbial dimethylarginine dimethylaminohydrolase enzymes. Mol Microbiol 1999;33:1278-1279.

17 Vallance P, Leiper J: Cardiovascular biology of the asymmetric dimethylarginine:dimethylarginine dimethylaminohydrolase pathway. Arterioscler Throm Vasc Biol 2004;24:1023-1030.

18 Yeni-Komshian H, Carantoni M, Abbasi F, Reaven GM: Relationship between several surrogate estimates of insulin resistance and quantification of insulin-mediated glucose disposal in 490 healthy nondiabetic volunteers. Diabetes Care 2000;23:171-175.

19 Stuhlinger MC, Abbasi F, Chu JW, Lamendola C, McLaughlin TL, Cooke JP, Reaven JM, Tsao PS: Relationship between insulin resistance and an endogenous nitric oxide synthase inhibitor. JAMA 2002;287:1420-1426.

20 Hardie DG, Hawley SA, Scott JW: AMP-activated protein kinase - development of the energy sensor concept. J Physiol 2006;574:7-15.

-21 Foretz M, Hebrard S, Leclerc J, Zarrinpashneh E, Soty M, Viollet B: Metformin inhibits hepatic gluconeogenesis in mice independently of the LKB1/AMPK pathway via a decrease in hepatic energy state. J Clin Invest 2010;120:2355-2369.

22 Kirpichnikov D, McFarlane S, Sowers JR: Metformin: an update. Ann Intern Med 2002;137:25-33.

23 Detaille D, Guigas B, Leverve X, Wiernsberger N, Devos P: Obligatory role of membrane events in the regulatory effect of metformin on the respiratory chain function. Biochem Pharmacol 2002;63:1259-1272.

24 Sud N, Wells SM, Sharma S, Wiseman DA, Wilham J, Black SM: Asymmetric dimethylarginine inhibits HSP90 activity in pulmonary arterial endothelial cells: role of mitochondrial dysfunction. Am J Physiol Cell Physiol 2008; 294:C1407-C1418.

25 Gruber HJ, Mayer C, Meinitzer A, et al: Asymmetric dimethylarginine (ADMA) is tightly correlated with growth in juveniles without correlations to obesity related disorders. Exp Clin Endocrinol Diabetes 2008;116:520-524.

26 Adcock IM, Ford P, Ito K, Barnes PJ: Epigenetics and airway disease. Respir Res 2006;7:21.

-27 Johannes F, Porcher E, Teixeira FK, et al: Assessing the impact of transgenerational epigenetic variation on complex traits. PLoS Genet 2009;5:e1000530.

28 Zakrzewicz D, Eickelberg O: From arginine methylation to ADMA: a novel mechanism with therapeutic potential in chronic lung diseases. BMC Pulm Med 2009;9:5.

29 Villeneuve LM, Reddy MA, Lanting LL, et al: Epigenetic histone H3 lysine 9 methylation in metabolic memory and inflammatory phenotype of vascular smooth muscle cells in diabetes. Proc Natl Acad Sci USA 2008;105:9047-9052.

30 Smith CL, Anthony S, Hubank M, et al: Effects of ADMA upon gene expression: an insight into the pathophysiological significance of raised plasma ADMA. PLoS Med 2005;10:1031-1043.

- 31 Wells SM, Holian A: Asymmetric dimethylarginine induces oxidative and nitrosative stress in murine lung epithelial cells. Am J Respir Cell Mol Biol 2007;36(5):520-528. 


\section{CardioRenal Medicine}

Cardiorenal Med 2011;1:211-219

\begin{tabular}{l|l}
\hline DOI: $10.1159 / 000332382$ & $\begin{array}{l}\text { C } 2011 \text { S. Karger AG, Basel } \\
\text { www.karger.com/crm }\end{array}$ \\
\hline
\end{tabular}

Bestermann: The ADMA-Metformin Hypothesis

-32 Ding G, Zhang A, Huang S: Ang II induces c-Jun NH2-terminal kinase activation and proliferation of human mesangial cells via redox-sensitive transactivation of the EGFR. Am J Physiol Renal Physiol 2007;293:F1889-F1897.

-33 Chen MF, Xie XM, Yang TL, et al: Role of asymmetric dimethylarginine in inflammatory reactions by angiotensin II. J Vasc Res 2007;44:391-402.

34 Sowers JR, Whaley-Connell A, Epstein M: Narrative review: the emerging clinical implications of the role of aldosterone in the metabolic syndrome and resistant hypertension. Ann Intern Med 2009;90:776-783.

- 35 Huang S, Zhang A, Ding G, et al: Aldosterone-induced mesangial cell proliferation is mediated by EGF receptor transactivation. Am J Physiol Renal Physiol 2009;296:F1323-F1333.

- 36 Motoshima J, Goldstein BJ, Igata M, et al: AMPK and cell proliferation - AMPK as a therapeutic target for atherosclerosis and cancer. J Physiol 2006;574:63-71.

- 37 Um SH, D’Alessio D, Thomas G: Nutrient overload, insulin resistance, and ribosomal protein S6 kinase 1, S6K1. Cell Metab 2006;3:393-402.

- 38 Bouley A, Zumstein-Mecker S, Stephan C, Benvink L, Lane HA: Antitumor efficacy of intermittent treatment schedules with the rapamycin derivative RAD101 correlates with prolonged inactivation of ribosomal protein 6 kinase in peripheral blood mononuclear cells. Cancer Res 2004;64:252-261.

-39 Yang ZC, Wang KS, Wu Y, et al: Asymmetric dimethylarginine impairs glucose utilization via ROS/TLR4 pathway in adipocytes: an effect prevented by vitamin E. Cell Physiol Biochem 2009;24:115-124.

- 40 Eskan MA, Benakanakere MR, Rose BG, Zhang P, Zhao J, Stathopoulou P, Fujioka D, Kinane DF: Interleukin-1ß modulates proinflammatory cytokine production in human epithelial cells. Infect Immun 2008;76:2080-2089.

-41 Calvert JW, Gundewar S, Jha S, Greer JJM, Bestermann WH, Tian R, Lefer DJ: Acute metformin therapy confers cardioprotection against myocardial infarction via AMPK-eNOS-mediated signaling. Diabetes 2008;57:696-705.

42 Marchetti P, Del Guerra S, Marselli L, et al: Pancreatic islets from type 2 diabetic patients have functional defects and increased apoptosis that are ameliorated by metformin. J Clin Endocrinol Metab 2004;89:5535-5541.

-43 Isoda K, Young JL, Zirlik A, et al: Metformin inhibits proinflammatory responses and nuclear factor-кB in human vascular wall cells. Arterioscler Thromb Vasc Biol 2006;26:611-617.

-44 Kalender A, Selvari A, Kim S, et al: Metformin independent of AMPK inhibits mTORC1 in a Rag GTPase-dependent manner. Cell Metab 2010;11:390-401.

-45 Zhuang Y, Miskimins WK: Cell cycle arrest in metformin treated breast cancer cells involves activation of AMPK, downregulation of cyclin D1, and requires p27Kip1 or p21Cip1. J Mol Signal 2008;3:18. 